\title{
Correction to: Disorders of eating and body image during the menopausal transition: associations with menopausal stage and with menopausal symptomatology
}

\author{
Barbara Mangweth-Matzek ${ }^{1}\left[\right.$ ] Claudia Ines Rupp ${ }^{2}$. Sophia Vedova ${ }^{1} \cdot$ Vanessa Dunst $^{1} \cdot$ Philine Hennecke $^{1}$. \\ Martin Daniaux ${ }^{3} \cdot$ Harrison G. Pope ${ }^{4}$
}

Published online: 5 March 2022

C) Springer Nature Switzerland AG 2022

\section{Correction to: \\ Eating and Weight Disorders - Studies on Anorexia, Bulimia and Obesity (2021) 26: 2763-2769 https://doi.org/10.1007/s40519-021-01141-4}

The first sentence of the results in the abstract section had been incorrectly published in the original publication. The complete correct result section is given below.

Results: The women as a whole reported a substantial prevalence of eating pathology, with 28 (12.4\%) displaying eating disturbances as reflected by a Global Score of $\geq 2.3$ on the Eating Disorder Examination Questionnaire (EDE-Q). Women in each of the three individual menopausal stages (premenopausal, perimenopausal, and postmenopausal) did not differ significantly from one another in eating and bodyimage measures. However, MRS scores showed strong associations with most measures of the Eating Disorder Examination Questionnaire, as well as with questions regarding satisfaction with body image. These associations remained little changed even when removing the four psychological

The original article can be found online at https://doi.org/10.1007/ s40519-021-01141-4.

Barbara Mangweth-Matzek

barbara.mangweth@i-med.ac.at

1 Department of Psychiatry, Psychotherapy and Psychosomatics, University Hospital of Psychiatry II, Medical University Innsbruck, Innsbruck, Austria

2 Department of Psychiatry, Psychotherapy and Psychosomatics, University Hospital of Psychiatry I, Medical University, Innsbruck, Austria

3 Breast Diagnostic Unit, Department of Radiology, University Hospital Innsbruck, Innsbruck, Austria

4 Biological Psychiatry Laboratory, Harvard Medical School, McLean Hospital, Massachusetts, USA items from the MRS score and examining only the association of the MRS somato-vegetative and urogenital items with these outcome variables.

Publisher's Note Springer Nature remains neutral with regard to jurisdictional claims in published maps and institutional affiliations. 